\title{
Systematic characterization of the ubiquitin-specific proteases in Magnaporthe oryzae
}

\author{
Xuan Cai', Zhao Wang ${ }^{1}$, Yuxi Hou', Caiyun Liu', Ahmed Hendy ${ }^{1,2}$, Junjie Xing ${ }^{3}$ and Xiao-Lin Chen ${ }^{1,3^{*}}$
}

\begin{abstract}
Ubiquitin-specific protease (UBP) family is the largest group of deubiquitinases, which plays important roles in eukaryotic organisms. Comprehensive analysis of UBP genes has not been conducted in the plant pathogenic fungi. In this study, 11 putative UBP genes were identified and characterized in the rice blast fungus Magnaporthe oryzae. Expression profile analysis showed that UBP3, UBP6, UBP12 and UBP14 were highly expressed in different tissues of M. oryzae. In all ubp mutants, especially $\Delta u b p 3, \Delta u b p 12$ and previously reported $\Delta u b p 14$, the ubiquitination levels were evidently elevated, which is consistent with their molecular roles in de-ubiquitination. The $\Delta u b p 1, \Delta u b p 3$, $\Delta u b p 4, \Delta u b p 8$ and $\Delta u b p 14$ mutants were reduced in colony growth. Most of the ubp mutants were severely reduced in conidia production capacity, indicating important roles of the UBPs in conidia formation. Except for $\triangle u b p 2$ and $\Delta u b p 16$, all of the other mutants were decreased in virulence to host plants and defective in invasive growth. These ubp mutants also induced massive ROS accumulation in host cells. We also found that the UBPs may function as both positive and negative regulators in stress response and nutrient utilization of $M$. oryzae. Collectively, UBPs are important for development, stress response, nutrient utilization and infection of M. oryzae.
\end{abstract}

Keywords: Ubiquitin, Deubiquitinases, Ubiquitin-specific proteases, Infection process, Gene family, Magnaporthe oryzae

\section{Background}

Ubiquitination is one of the most important posttranslational modifications, which plays central roles in diverse cellular pathways such as cell-cycle progression, signal transduction, DNA repair and endocytosis and apoptosis (Hershko and Ciechanover 1998; Pickart and Eddins 2004; Dikic et al. 2009). The ubiquitin molecules are catalyzed by a cascade of three enzymes: the ubiquitin activating enzyme (E1), the ubiquitin conjugating enzyme (E2) and the ubiquitin ligase (E3) (Pickart and Eddins 2004). The fate of ubiquitinated substrate proteins

\footnotetext{
* Correspondence: chenxiaolin@mail.hzau.edu.cn

'The Provincial Key Lab of Plant Pathology of Hubei Province, College of Plant Science and Technology, Huazhong Agricultural University, Wuhan 430070, China

${ }^{3}$ State Key Laboratory of Hybrid Rice, Hunan Hybrid Rice Research Center, Changsha 410082, China

Full list of author information is available at the end of the article
}

mainly depends on the number of conjugated ubiquitin and the type of linkage. Proteins linked by Lys48 bearing four or more ubiquitin chains are mostly recognized by the $26 \mathrm{~S}$ proteasome complex system for degradation. Attachment of ubiquitin to cellular proteins as different linkage types are involved in distinct cellular processes (Pickart and Eddins 2004).

As a reversible event, ubiquitins can be removed from proteins, which is also important for regulation of different cellular processes. Cellular ubiquitin monomers are kept in homeostasis by processing from ubiquitin precursors or deubiquitinating from protein substrates (Amerik and Hochstrasser 2004; Nijman et al. 2005). These processes are regulated by the de-ubiquitinating enzymes (DUBs), which can specifically remove ubiquitin moiety. The cleavage of ubiquitin from proteins by DUBs can affect the activity and fate of substrates (Wilkinson 1997;

(c) The Author(s). 2020 Open Access This article is licensed under a Creative Commons Attribution 4.0 International License, which permits use, sharing, adaptation, distribution and reproduction in any medium or format, as long as you give appropriate credit to the original author(s) and the source, provide a link to the Creative Commons licence, and indicate if changes were made. The images or other third party material in this article are included in the article's Creative Commons licence, unless indicated otherwise in a credit line to the material. If material is not included in the article's Creative Commons licence and your intended use is not permitted by statutory regulation or exceeds the permitted use, you will need to obtain permission directly from the copyright holder. To view a copy of this licence, visit http://creativecommons.org/licenses/by/4.0/. 
Amerik and Hochstrasser 2004; Crosas et al. 2006). Among several types of DUBs, the ubiquitin-specific proteases (UBPs) are highly conserved and the largest subfamily among eukaryotic organisms (Wilkinson 1997; Amerik and Hochstrasser 2004; Nijman et al. 2005), which commonly possess a triad of catalytic residues in conserved cysteine and histidine boxes (Hu et al. 2002). Ubiquitin C-terminal hydrolases (UCHs) contain a similar triad of catalytic residues in two conserved cysteine and histidine boxes (Johnston et al. 1999; Amerik and Hochstrasser 2004). Other DUBs include the ovarian tumor proteases (OTUs), Machado-Joseph disease protein domain proteases (MJDs), etc. (Balakirev et al. 2003; Burnett et al. 2003; Scheel et al. 2003; Nanao et al. 2004).

There are 16 UBP genes in Saccharomyces cerevisae (Wilkinson 1997) and 27 UBP genes in Arabidopsis thaliana (Liu et al. 2008). Studies on S. cerevisae revealed UBPs are involved in nutrient utilization, energy metabolism, sexual reproduction, and stress responses (Kahana 2001; Enyenihi and Saunders 2003; Dudley et al. 2005; Auesukaree et al. 2009). In plants, UBPs have been proved to play roles in cell proliferation, root hair elongation, mitochondria morphogenesis, pollen development and transmission, and abscisic acid (ABA)-mediated resistance to salt and drought stress, etc. (Doelling et al. 2001; Liu et al. 2008; Du et al. 2014; Xu et al. 2016; Zhao et al. 2016). However, few studies have addressed systematic analysis on the roles of all members of the $U B P$ gene family, although roles of single $U B P$ gene was reported (Liu et al. 2008; Wu et al. 2019).

Magnaporthe oryzae is a fungus threatening rice production worldwide, and also a model fungal plant pathogen (Wilson and Talbot 2009; Yan and Talbot 2016). This fungus forms a specialized infection structure appressorium on the host surface, and penetrates into host cells (de Jong et al. 1997; Dixon et al. 1999; Thines et al. 2000). In the host cells, the fungus forms invasive hyphae to establish biotrophic colonization and eventually converts into necrotrophic growth for spreading (Kankanala et al. 2007). Previous study has found that both $M$. oryzae UBP14 and UBP4 play key roles in growth, conidiation, stress response, and carbon sources utilization, and meanwhile, are required for virulence of the pathogen (Wang et al. 2018; Que et al. 2019). In order to systematically determine functions of UBPs, we identified and characterized roles of all $M$. oryzae UBP genes in development, stress response, carbon source utilization, as well as the virulence.

\section{Results}

Identification of the UBP family proteins in M. oryzae

To carry out a genome-wide identification of the UBP protein family in $M$. oryzae, S. cerevisiae UBP protein sequences were used as queries to search against the protein database available in the Magnaporthe genome
(http://fungi.ensembl.org/Magnaporthe_oryzae/Info/ Index). We identified 11 MoUBP candidate proteins, which were used to analyze the conserved domain using the Pfam database (http://pfam.xfam.org). MoUBP proteins exhibited a complete $\mathrm{UCH}$ domain with two short but well-conserved motifs specific to UBP proteins (Cys and His) (Amerik and Hochstrasser 2004). They were named UBP1 (MGG_04031T0), UBP2 (MGG_00877T0), UBP3 (MGG_05996T0), UBP4 (MGG_04957T0), UBP6 (MGG_02970T0), UBP8 (MGG_03527T0), UBP12 (MGG_05666T0), UBP13 (MGG_09907T0), UBP14 (MGG_08270T0), UBP15 (MGG_05002T0) and UBP16 (MGG_04494T0), respectively. These UBP proteins also had some non-UBP domains, including RHOD (UBP4), UBQ (UBP6), ZF-UBP (UBP8 and UBP14), DUSP (UBP12), UIM (UBP13), DUF3517 (UBP15) and UBA (UBP14) domains (Fig. 1a).

To determine the phylogenetic relationship of the UBPs among different species, a Neighbor-joining phylogenetic tree was constructed based on the alignments of fulllength UBP protein sequences from S. cerevisiae, Aspergillus nidulans, Neurospora crassa, Fusarium graminearum and M. oryzae (Fig. 1b). In the phylogenetic tree, all sequences were classified into 11 groups. The phylogenetic dendrogram shows that, for most cases, $M$. oryzae UBP proteins are evenly distributed among these clades, implying that genes in the clade may have evolved from a copy of their common fungal ancestor (Fig. 1b).

\section{Expression patterns of UBP genes at different developmental stages of $M$. oryzae}

The gene expression pattern can provide vital clues for gene's function. To characterize the expression patterns of $U B P$ genes, we analyzed their transcription levels in different tissues, including mycelia, conidia, appressoria (at 3 hours post inoculation [hpi] or $12 \mathrm{hpi}$ ), and invasive hyphae (at 18, 24 or $42 \mathrm{hpi}$ ) according to an unpublished RNAseq data. As shown in Fig. 2, UBP3, UBP6, UBP12 and UBP14 showed high expression levels in nearly all tested tissues, while $U B P 1, U B P 13$ and $U B P 15$ showed low expression levels in these tissues. In particular, four genes $(U B P 3$, $U B P 6, U B P 12$ and $U B P 14)$ in mycelia, seven genes (UBP3, UBP4, UBP6, UBP8, UBP12, UBP14 and UBP16) in conidia, most of these genes in appressoria, and five genes (UBP3, UBP6, UBP12, UBP14 and UBP16) in invasive hyphae showed high expression levels. Taken together, $M$. oryzae UBP genes exhibited different expression profiles in different tissues, indicating diverse biological functions of $U B P$ genes in developmental and infection processes.

Single deletions of the UBP genes increase ubiquitination levels in M. oryzae

To characterize the functions of $U B P$ genes in $M$. ory$z a e$, single gene deletion mutant for each $U B P$ gene was 
a

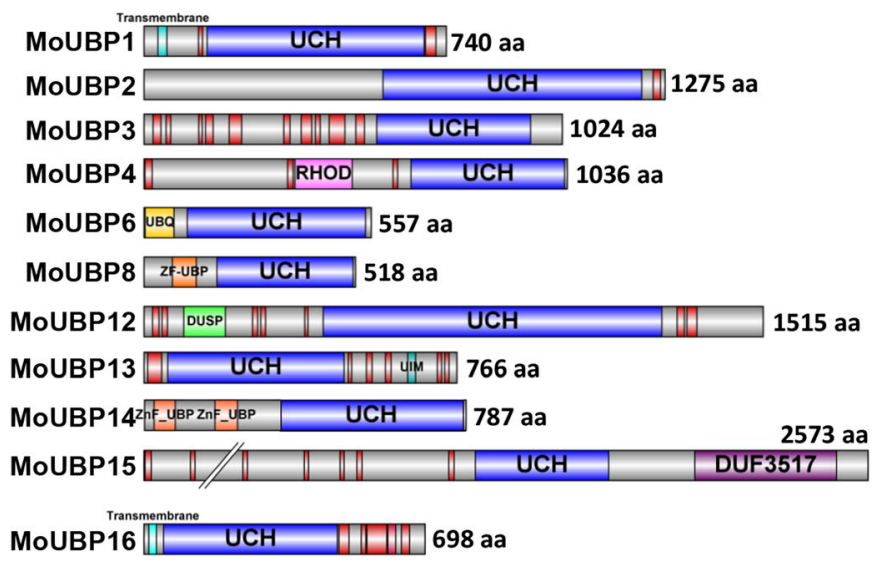

b

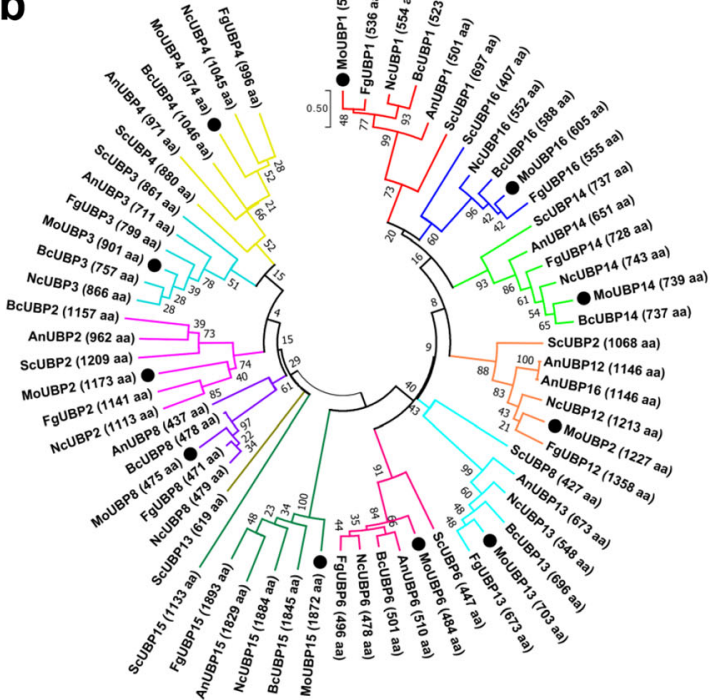

Fig. 1 The UBP family in M. oryzae. a Structures and domains of the UBP proteins in M. oryzae. UCH, ubiquitin C-terminal hydrolases; RHOD, rhodanese homology domain; UBQ, ubiquitin homologues; ZF-UBP, Zn-finger in ubiquitin-hydrolases; DUSP, domain present in ubiquitin-specific protease; UIM, ubiquitin-interacting motif; DUF, domains of unknown function; UBA, ubiquitin-associated domain. $\mathbf{b}$ Phylogenetic analyses of putative UBP proteins in fungi. The phylogenetic tree was constructed using MEGA7.0 by neighbour-joining method. The Bootstrap replicates were 1000. The sequences were collected from organisms as follows: Sc, Saccharomyces cerevisiae; An, Aspergillus nidulans; Nc, Neurospora crassa; Mo, Magnaporthe oryzae; Fg, Fusarium graminearum; Bc, Botrytis cinerea. The proteins IDs of MoUBPs are shown as below: MoUBP1 (MGG 04031), MoUBP2 (MGG_00877), MoUBP3 (MGG_05996), MoUBP4 (MGG_04957), MoUBP6 (MGG_02970), MoUBP8 (MGG_03527), MoUBP12 (MGG_05666), MoUBP13 (MGG_09907), MoUBP14 (MGG_08270), MoUBP15 (MGG_05002) and MoUBP16 (MGG_04494)

generated by using a split-PCR strategy (Goswami 2012). At least two independent null mutants with identical phenotypes were obtained for each UBP gene, and all deletion mutants were confirmed by polymerase chain reaction (PCR) and gene expression level detection
(Additional file 1: Figure S1 and Additional file 2: Figure S2).

In order to test whether deletion of single $U B P$ gene affects total ubiquitination level in $M$. oryzae, all of the single $u b p$ mutants (except for $\Delta u b p 14$ ) were tested for

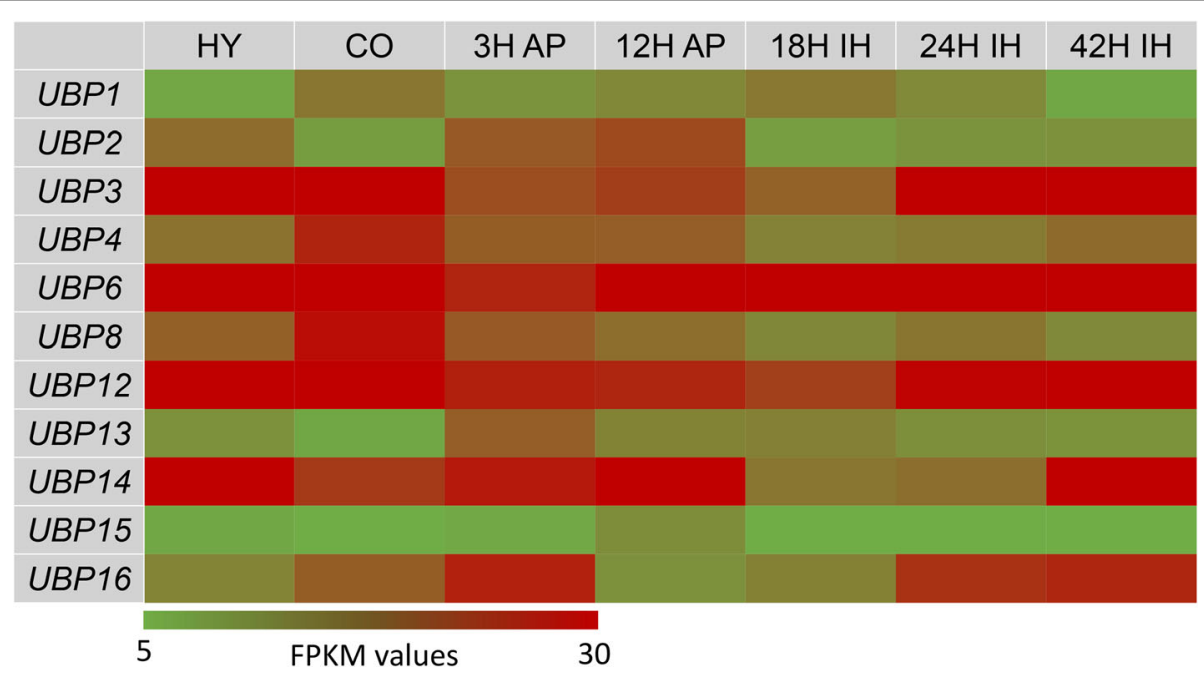

Fig. 2 The heat map shows expression patterns of UBP genes at different developmental stages. The expression level of each gene relative to the mean FPKM (Fragments Per Kilobase of transcript per Million mapped reads) value across all the experimental stages on the log scale has been represented. The color (from green to red) indicates gene expression intensity from low to high. HY: Mycelial hyphae; CO: Conidia; $3 \mathrm{H}$ AP: appressoria at $3 \mathrm{hpi} ; 12 \mathrm{H} \mathrm{AP}$ : appressoria at $12 \mathrm{hpi} ; 8 \mathrm{IH}$ : invasive hyphae at $18 \mathrm{hpi} ; 24 \mathrm{IH}$ : invasive hyphae at $24 \mathrm{hpi} ; 42 \mathrm{IH}$ : invasive hyphae at $42 \mathrm{hpi}$ 
changes of the ubiquitination level (Fig. 3). As expected, all of the mutants displayed increased ubiquitination levels. Especially, the $\Delta u b p 3$ and $\Delta u b p 12$ mutants, as well as our previously reported $\Delta u b p 14$ (Wang et al. 2018), each of them showed significant increase of the ubiquitination level compared to the wild type. The $\Delta u b p 4, \Delta u b p 8, \Delta u b p 13, \Delta u b p 15$ and $\Delta u b p 16$ mutants showed a moderate increase in ubiquitination level. These data reflected that all of the $U B P$ genes are indeed involved in deubiquitinating process in M. oryzae.

\section{Characterization of vegetative growth of the $u b p$ mutants} All of the $u b p$ mutants were examined for phenotypic changes in colony growth, mycelium formation, conidium formation, appressorium formation, and invasive growth. As summarized in Table 1, the deletion mutants of each UBP gene displayed distinctive effects on colony growth, conidiation, appressorium formation and infection-related morphogenesis. Among 11 mutants, $\Delta u b p 1, \Delta u b p 3, \Delta u b p 4, \Delta u b p 8$ and $\Delta u b p 14$ mutants displayed respectively $22.0 \%, 9.4 \%$, $6.1 \%, 12.7 \%$ and $25.3 \%$ reductions of colony diameter on oatmeal tomato agar (OTA) plates (Fig. 4a, b and Table 1). Correspondingly, significant reductions of mycelial cell length were observed in these mutants (Fig. 4c, d). These results indicated that $U B P 1, U B P 3$, $U B P 4, U B P 8$ and $U B P 14$ are involved in the regulation of vegetative growth in $M$. oryzae.

\section{Characterization of conidium production of the $u b p$ mutants}

Conidia play important roles in the disease cycle of the rice blast fungus. Most $U B P$ genes were found here to be involved in conidiation of $M$. oryzae. The $\Delta u b p 1$, $\Delta u b p 3, \Delta u b p 4, \Delta u b p 6, \Delta u b p 8, \Delta u b p 12, \Delta u b p 13, \Delta u b p 14$ and $\Delta u b p 16$ mutants were markedly reduced in conidiation compared with the wild type P131 strain (Fig. 5a and Table 1). For the $\Delta u b p 8$ and $\Delta u b p 14$ mutants, conidia were very rare with only few visible spores following examination of more than ten 10-day-old culture plates. However, the $\Delta u b p 2$ and $\Delta u b p 15$ mutants showed normal conidium production (Fig. 5a and Table 1). Accordingly, conidiophore formation of the $\Delta u b p 1$, $\Delta u b p 3, \Delta u b p 4, \Delta u b p 6, \Delta u b p 8, \Delta u b p 12, \Delta u b p 13, \Delta u b p 14$ and $\Delta u b p 16$ mutants were severely reduced (Fig. $5 \mathrm{~b}$ ). These results showed that most members of the $U B P$ genes regulate conidiation in $M$. oryzae.

Except for $\Delta u b p 8$, no other $u b p$ mutants were blocked in conidium germination, and most of them did not show significant changes in appressorium formation. The $\Delta u b p 8$ mutant displayed a lower percentage of appressorium formation compared with the wild-type strain and other $u b p$ mutants (Table 1).

\section{Virulence and infection process of the ubp mutants}

To further test whether these $M$. oryzae $U B P$ genes are involved in virulence, conidia of each of the $u b p$ mutants were sprayed onto susceptible rice seedlings. As shown in Fig. 6a, very few lesions were formed on the rice

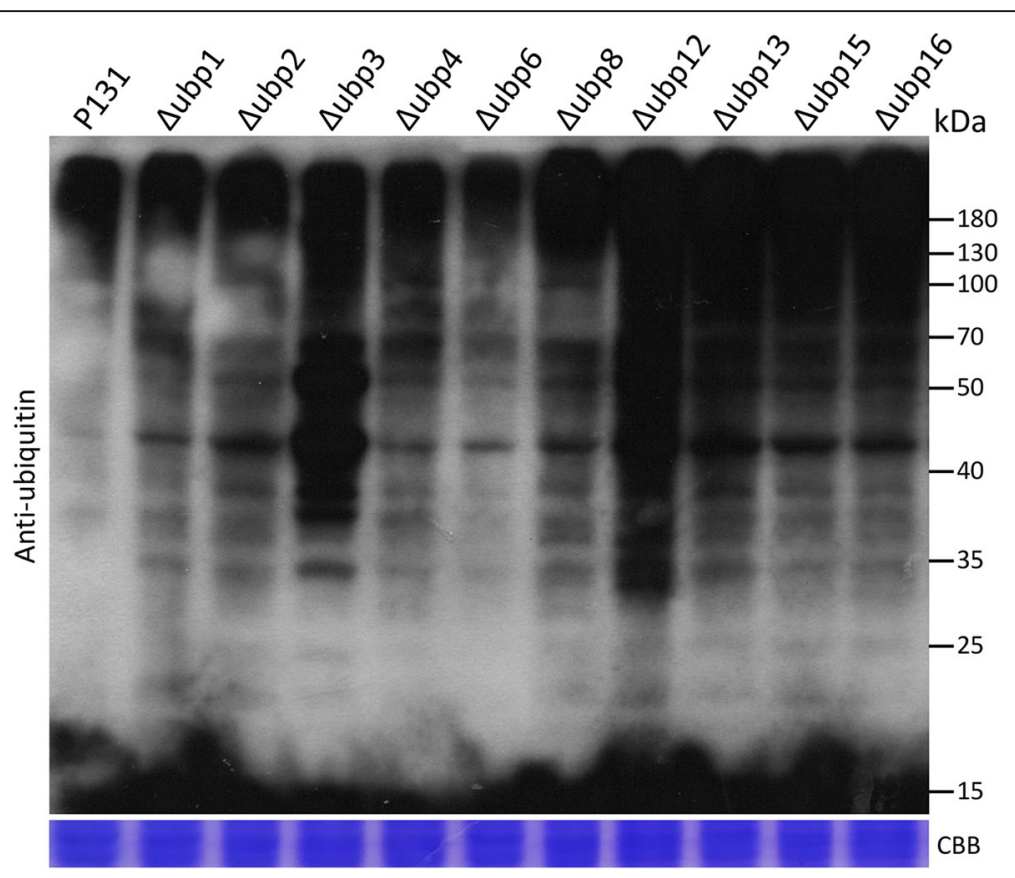

Fig. 3 Anti-ubiquitin immunoblot analysis of ubp mutants. A monoclonal antibody against ubiquitin was used (1:5000). The coomassie brilliant blue (CCB) stain was used for protein loading 
Table 1 Comparison of biological phenotypic characteristics among strains

\begin{tabular}{|c|c|c|c|}
\hline Strain & Growth $(\mathrm{cm})$ & Conidiation $\left(\times 10^{4} / \mathrm{mL}\right)$ & Appressorium formation (\%) \\
\hline p131 & $3.95 \pm 0.06^{\mathrm{A}}$ & $239.7 \pm 8.2^{\mathrm{A}}$ & $97 \pm 1.2^{\mathrm{A}}$ \\
\hline$\Delta u b p 1$ & $3.08 \pm 0.05^{F}$ & $105.3 \pm 4.4^{\mathrm{D}}$ & $88 \pm 2.1^{B}$ \\
\hline$\Delta u b p 2$ & $3.95 \pm 0.05^{\mathrm{A}}$ & $244.0 \pm 7.0^{A}$ & $96 \pm 1.5^{\mathrm{A}}$ \\
\hline$\triangle u b p 3$ & $3.58 \pm 0.05^{\mathrm{D}}$ & $49 \pm 5.8^{E}$ & $88 \pm 4.5^{B}$ \\
\hline$\Delta u b p 4$ & $3.71 \pm 0.08^{C}$ & $146.7 \pm 9.2^{C}$ & $80 \pm 2.0^{c}$ \\
\hline$\Delta u b p 6$ & $3.89 \pm 0.06^{\mathrm{AB}}$ & $148.3 \pm 2.8^{C}$ & $88 \pm 4.0^{B}$ \\
\hline$\Delta u b p 8$ & $3.45 \pm 0.05^{\mathrm{E}}$ & $3.7 \pm 0.5^{G}$ & $62 \pm 4.0^{\mathrm{E}}$ \\
\hline$\Delta u b p 12$ & $3.85 \pm 0.05^{\mathrm{AB}}$ & $104.7 \pm 3.7^{\mathrm{D}}$ & $87 \pm 2.3^{B}$ \\
\hline$\Delta u b p 13$ & $3.90 \pm 0.06^{\mathrm{A}}$ & $169.3 \pm 3.3^{B}$ & $95 \pm 1.2^{\mathrm{A}}$ \\
\hline$\Delta u b p 14$ & $2.95 \pm 0.05^{G}$ & $26.7 \pm 0.7^{F}$ & $80 \pm 2.8^{C}$ \\
\hline$\Delta u b p 15$ & $3.95 \pm 0.05^{\mathrm{A}}$ & $243.3 \pm 6.6^{A}$ & $91 \pm 3.1^{\mathrm{AB}}$ \\
\hline$\Delta u b p 16$ & $3.95 \pm 0.05^{\mathrm{A}}$ & $144.3 \pm 5.8^{C}$ & $81 \pm 1.2^{c}$ \\
\hline
\end{tabular}

seedlings inoculated with $\Delta u b p 1, \Delta u b p 3, \Delta u b p 4, \Delta u b p 6$, $\Delta u b p 8$ and $\Delta u b p 14$ mutants. In contrast, rice seedlings sprayed with $\Delta u b p 2$ and $\Delta u b p 16$ mutants developed numerous typical rice blast lesions similar to that inoculated with the wild-type M. oryzae strain (Fig. 6a). When inoculating the mycelial agar plugs onto the wounded rice leaves, all of the virulence-decreased mutants produced evidently restricted spreading of the lesions (Fig. $6 \mathrm{~b})$. This result indicated that $U B P 1, U B P 3, U B P 4$, $U B P 6, U B P 8$ and $U B P 14$ play key roles in virulence to host. Since the $\Delta u b p 1, \Delta u b p 3, \Delta u b p 4, \Delta u b p 6, \Delta u b p 8$ and $\Delta u b p 14$ mutants exhibited normal appressoria formation, we examined the ability of the appressoria in the penetration of the barley epidermal cells. At $24 \mathrm{hpi}$, only $\Delta u b p 14$ showed severe reduction of penetration, while no evident changes were found for the other $u b p$ mutants, with majority of the appressoria in these mutants forming invasive hyphae (Fig. 6c). However, at both $24 \mathrm{hpi}$ and $30 \mathrm{hpi}$, the formation of infection hyphaein the $\Delta u b p 1, \Delta u b p 3, \Delta u b p 4, \Delta u b p 6, \Delta u b p 8$ and $\Delta u b p 14$ mutants was significantly retarded, compared with the wild-type strain (Fig. 6c). These results indicate that $U B P 1, U B P 3, U B P 4, U B P 6, U B P 8$ and $U B P 14$ regulate virulence of $M$. oryzae through affecting infection hyphal growth.

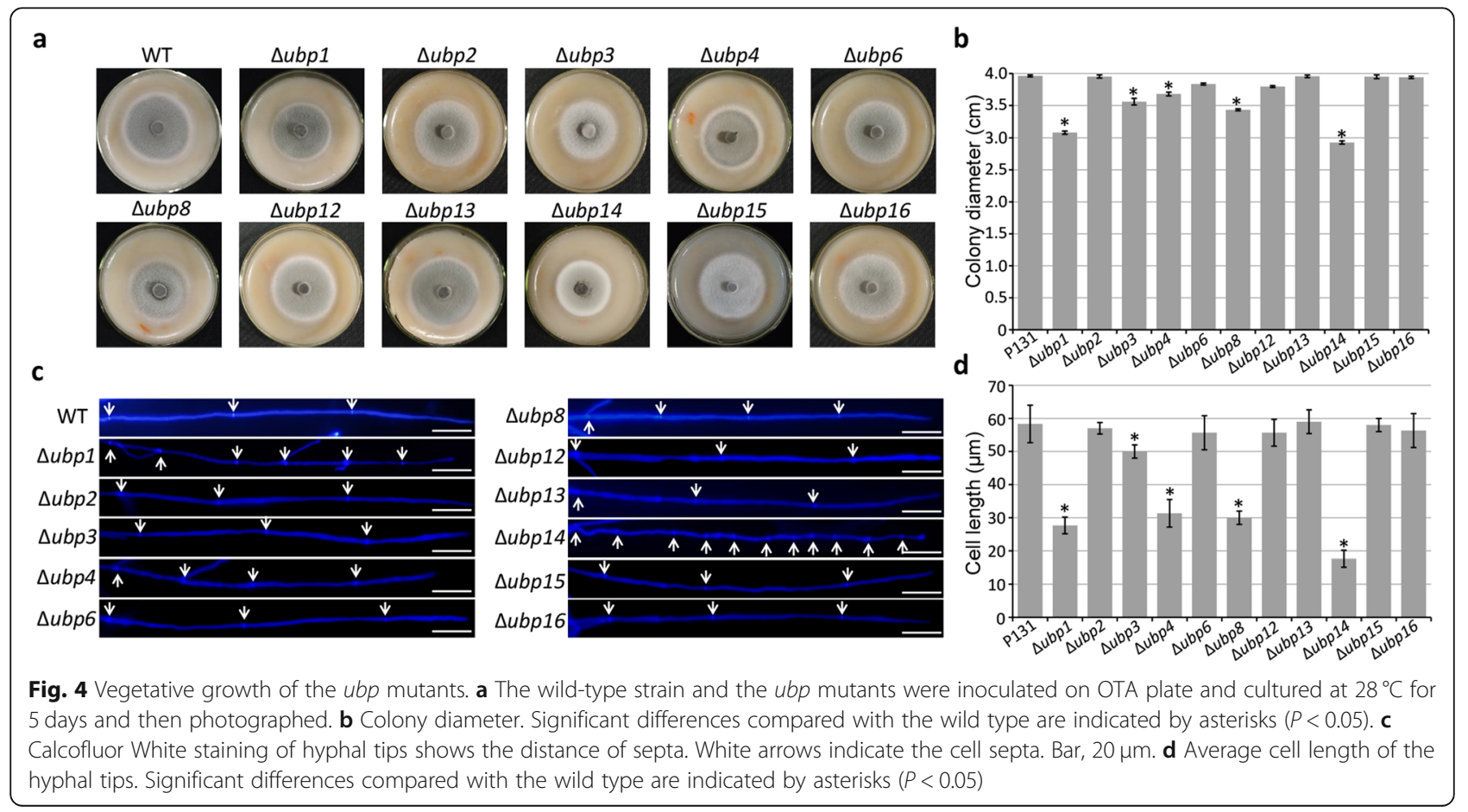


a

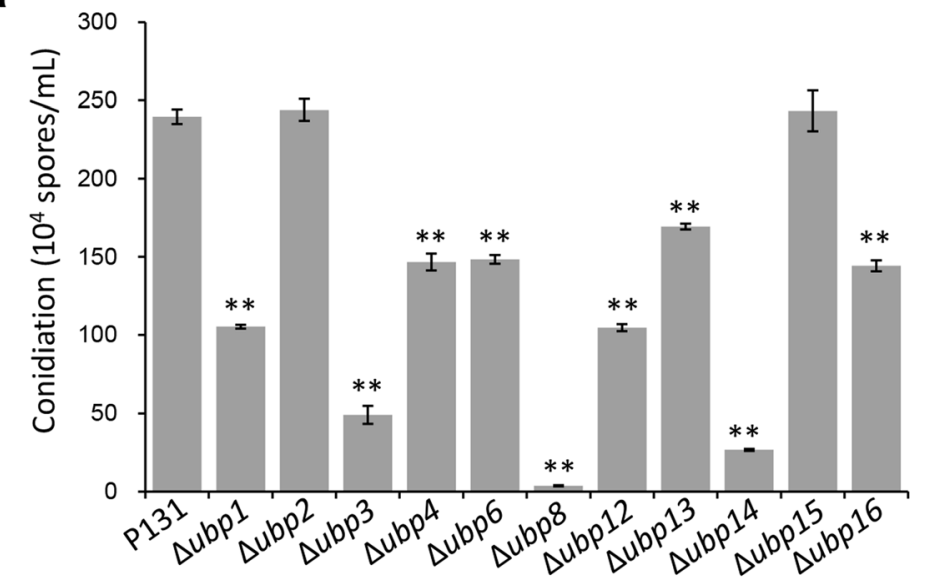

b

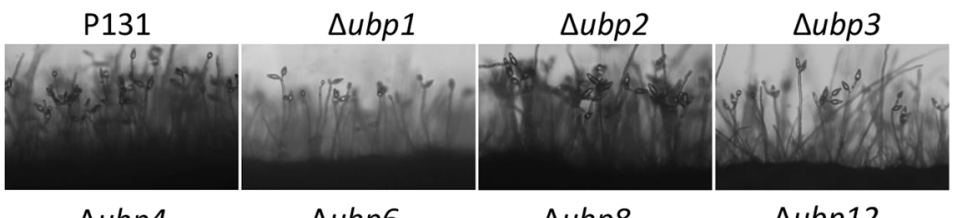

$\Delta u b p 4$

$\Delta u b p 6$

$\Delta u b p 8$

$\Delta u b p 12$
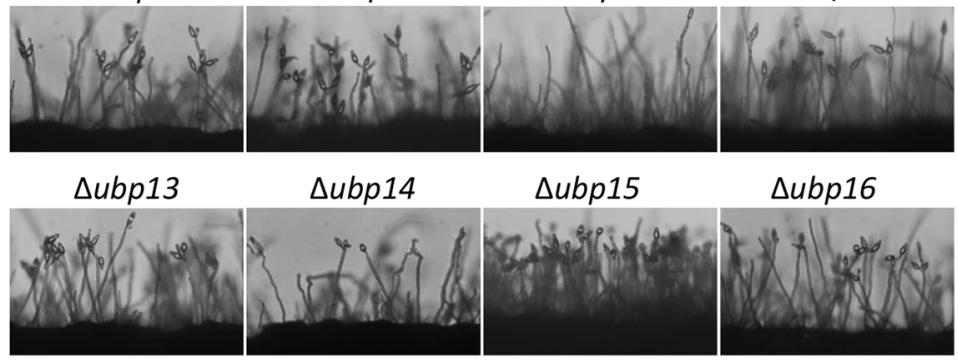

Fig. 5 Asexual development of the ubp mutants. a Conidiation capacity. Conidia were collected from strains growing on OTA plates $(\Phi=6 \mathrm{~cm})$. Means and standard errors were calculated from three independent experiments $(n>100)$. Significant differences compared with the wild type are indicated by asterisks $(P<0.01)$. b Conidiophore development was observed under light microscopy

The ubp mutants led to accumulation of host reactive oxygen species (ROS)

Because some $u b p$ mutants were blocked in host invasive growth, we explored whether each of these $U B P$ gene deletion mutants can result in host ROS accumulation. ROS accumulation in the barley epidermis cells infected by each strain at $30 \mathrm{hpi}$ was detected by staining with 3,3'-diaminobenzidine (DAB). The result demonstrated that, except for $\triangle u b p 2$ and $\Delta u b p 16$, the barley epidermis cells infected by all other $u b p$ mutants can be easily stained by $\mathrm{DAB}$, which were detected with abundant reddish-brown precipitates in the infected host cells (Fig. 7a, b). At the same time, host cells infected by the wild-type strain were not well stained by DAB (Fig. $7 \mathrm{a}, \mathrm{b})$. These results showed that $U B P$ genes are important for $M$. oryzae to respond to host cellular oxidative stress, and therefore required for invasive growth.
Members of UBP genes are involved in stress response in

\section{M. oryzae}

We evaluated the effect of $U B P$ genes disruption on stress tolerance of $M$. oryzae. The wild-type and $u b p$ mutant stains were inoculated onto the CM plates each containing different stresses, including the cell wall stresses $(0.1 \mathrm{mg} / \mathrm{mL}$ Calcofluor White [CFW], $0.2 \mathrm{mg} /$ $\mathrm{mL}$ Congo Red [CR] or $0.005 \%$ sodium dodecyl sulfate [SDS]), osmotic stress $(0.5 \mathrm{M} \mathrm{NaCl}$ or $1 \mathrm{M}$ sorbitol), or oxidative stress $\left(10 \mathrm{mM} \mathrm{H}_{2} \mathrm{O}_{2}\right)$. The results showed that, compared with the wild-type strain, the $\Delta u b p 1, \Delta u b p 3$, $\Delta u b p 8, \Delta u b p 12$ and $\Delta u b p 14$ mutants were more sensitive to cell wall perturbing reagents; the $\Delta u b p 8, \Delta u b p 12$ and $\Delta u b p 14$ mutants were more sensitive to osmotic stress; and the $\Delta u b p 3, \Delta u b p 12$ and $\Delta u b p 14$ mutants were more sensitive to oxidative stress (Fig. 8). Interestingly, we also noticed some mutants were more resistant to different stresses. For example, the $\Delta u b p 2$ and $\Delta u b p 4$ mutants were more resistant to cell wall stresses; the $\Delta u b p 1, \Delta u b p 2$ and $\Delta u b p 4$ mutants were more resistant 


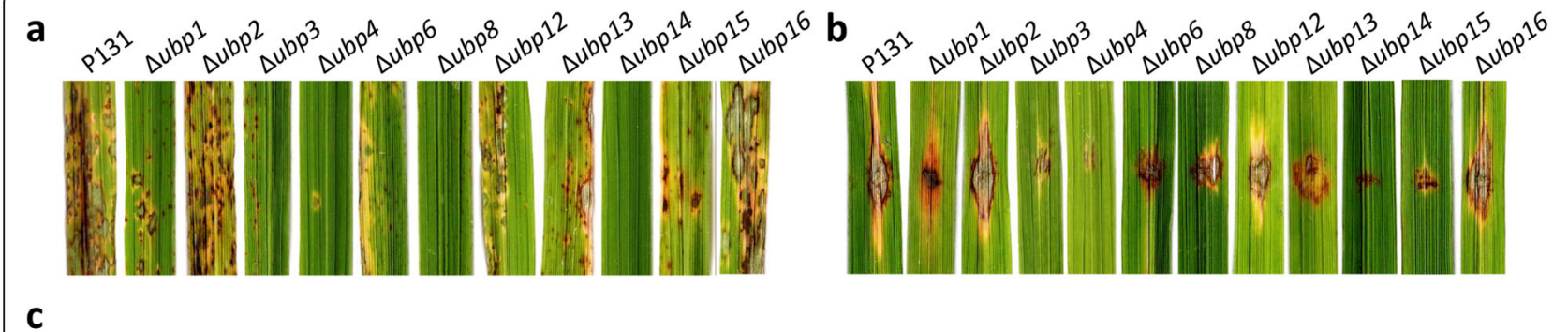

C
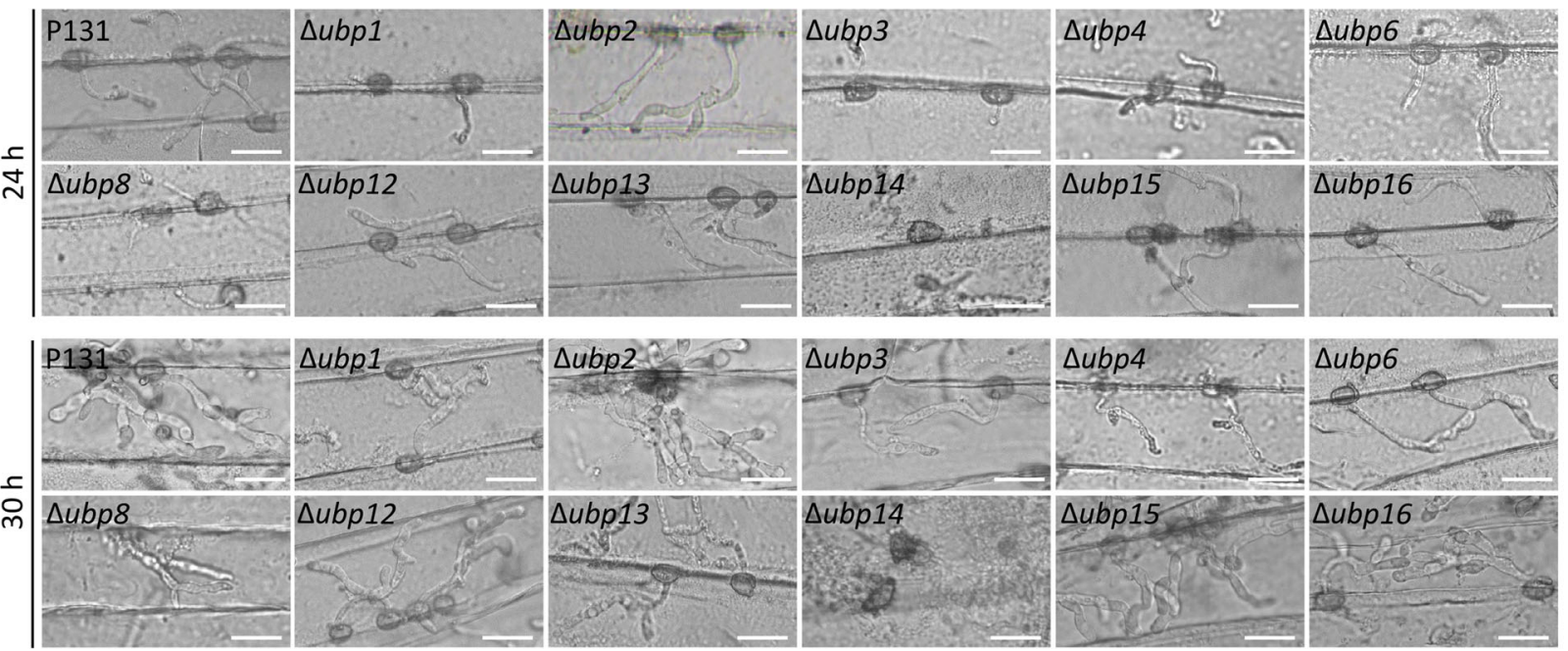

Fig. 6 Pathogenesis of the $u b p$ mutants. a Lesions formed on rice leaves sprayed with conidia of the wild-type and ubp mutant strains at 5 dpi. b Lesions formed on the wounded rice leaves inoculated with mycelial agar plugs of the wild-type and ubp mutant strains. c Invasive hyphae formed in barley epidermal cells at $24 \mathrm{hpi}$ and $30 \mathrm{hpi}$. Bar, $20 \mu \mathrm{m}$

to oxidative stress (Fig. 8). These data indicated that $U B P$ genes positively or negatively regulate response to different stresses in M. oryzae.

\section{Nutrient utilization was positively or negatively regulated by different UBP genes}

We also evaluated whether the disruption of $U B P$ genes can affect carbon source utilization of $M$. oryzae. The wild-type and $u b p$ mutant strains were cultured on MM plates supplemented with each of glucose, NaAc, ethanol and glycerol as the sole carbon source. After 5 days culture at $28{ }^{\circ} \mathrm{C}$, colony diameters of the $\Delta u b p 4$ and $\Delta u b p 14$ mutants were significantly reduced when grown on all of these conditions compared with those grown on the CM plates (Fig. 9). These demonstrated that the carbon sources utilization ability of the $\Delta u b p 4$ and $\triangle u b p 14$ mutants were significantly reduced, suggesting UBP4 and UBP14 are positive regulators of carbon source utilization. In contrast, the $\triangle u b p 1$ was significantly increased in growth in the NaAc and ethanol conditions, and the $\Delta u b p 8$ mutant was increased in growth in all tested conditions (Fig. 9), suggesting $U B P 1$ and UBP8 are negative regulators of carbon source utilization. Therefore, nutrient utilization of $M$. oryzae can be either positively or negatively regulated by different $U B P$ genes.

\section{Discussion}

As one of the largest families of DUBs, the eukaryoticspecific UBP family protein plays important roles in fungal growth and development. Detailed characteristics and functions of $U B P$ genes have been reported in S. cerevisae (Amerik et al. 2000), but not been systematically described in plant pathogenic fungi. Here, we identified and characterized 11 putative $U B P$ genes in the rice blast fungus $M$. oryzae using a genome-wide analysis.

The expression profiles of the $U B P$ genes at different developmental stages of $M$. oryzae have been evaluated. According to the gene expression patterns, different UBP proteins may have diverse functions. The $U B P 3$, $U B P 6, U B P 12$ and $U B P 14$ were highly expressed at different stages, suggesting they might play important roles at these stages. Moreover, some genes showed similar expression patterns in different tissues, suggesting that they might have retained redundant functions in regulating the same biological functions.

In fungi, functions of the $U B P$ genes have been revealed in yeast and other fungi including $M$. oryzae 


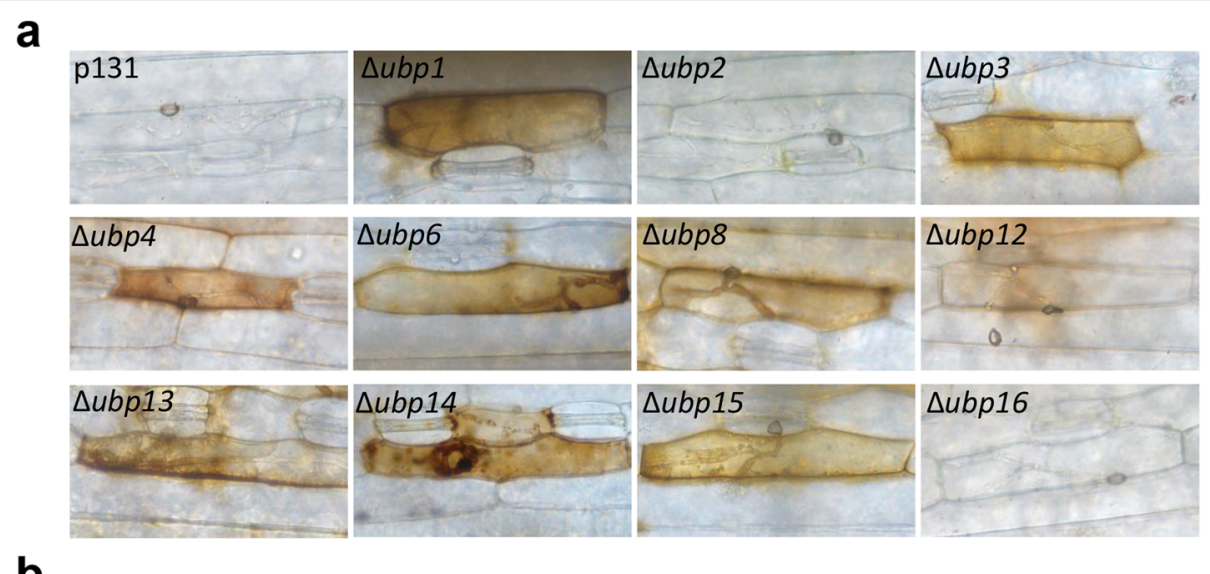

b

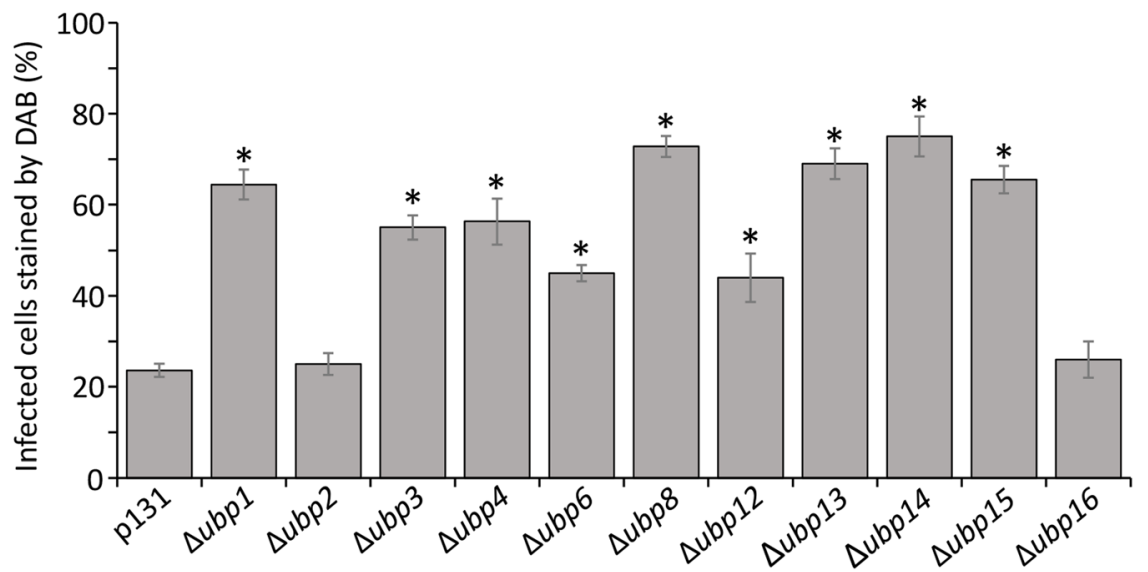

Fig. 7 Detection of the host cell ROS accumulation. a DAB-staining assays of the wild type and ubp mutants-infected barley epidermis cells. $\mathbf{b}$ Percentage of DAB-stained barley epidermis cells infected by the wild-type and ubp mutant strains. Means and standard errors were calculated from three independent replicates. Significant differences were indicated by asterisks $(P<0.05)$

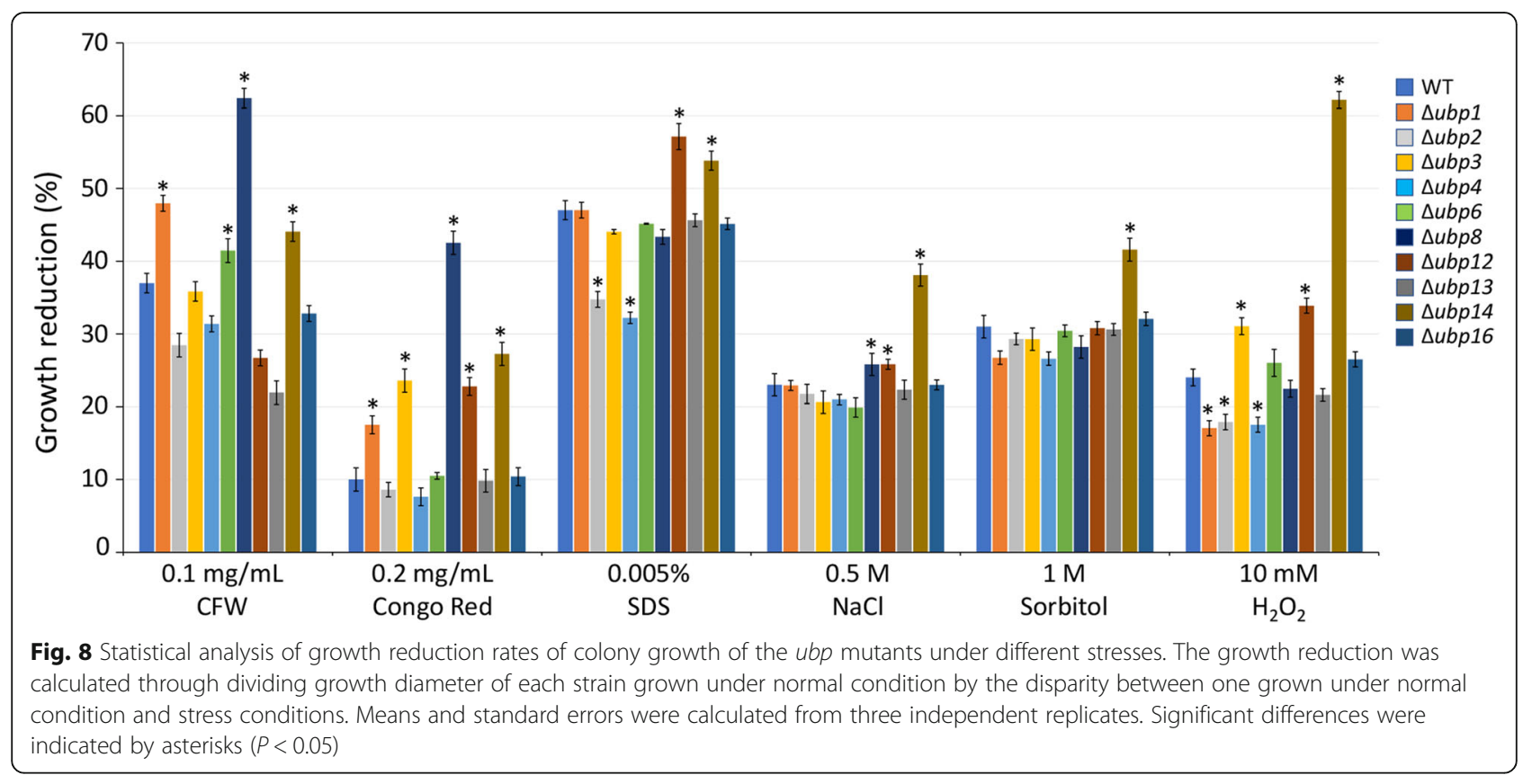




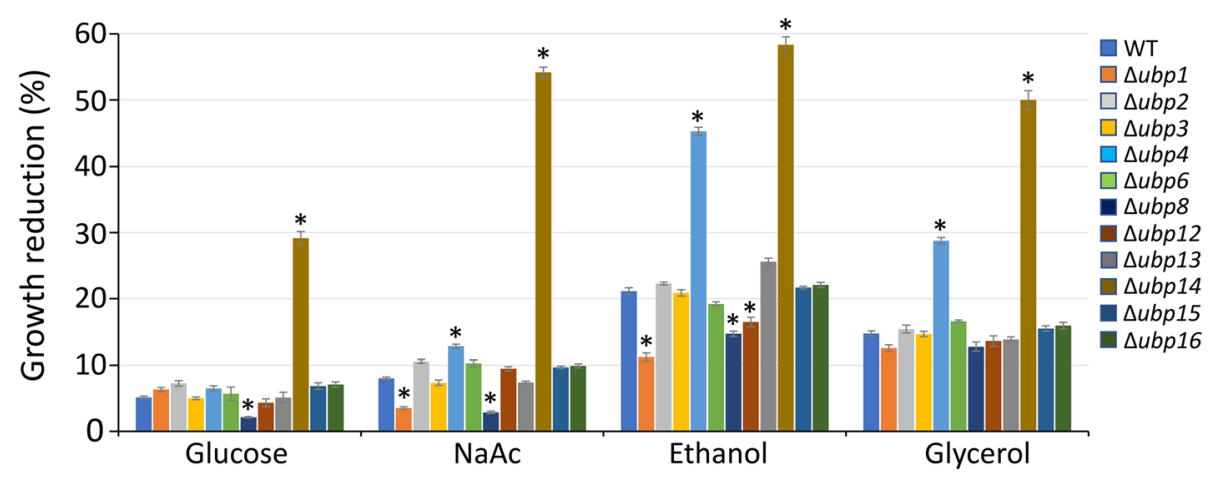

Fig. 9 Statistical analysis of growth reduction rates of colony growth on different carbon sources compared to growth on CM plates. The wildtype and ubp mutant stains cultured on CM or minimal medium agar containing 1\% glucose, $50 \mathrm{mM}$ sodium acetate, $50 \mathrm{mM}$ ethanol, or $50 \mathrm{mM}$ glycerol for $120 \mathrm{~h}$ at $28^{\circ} \mathrm{C}$. Means and standard errors were calculated from three independent replicates. Significant differences were indicated by asterisks $(P<0.01)$

(Amerik et al. 2000; Wang et al. 2018; Que et al. 2019). Sixteen $U B P$ genes are found in S. cerevisae to be involved in nutrient utilization, energy metabolism, sexual reproduction, and stress responses (Kahana 2001; Enyenihi and Saunders 2003; Dudley et al. 2005; Auesukaree et al. 2009). For example, $D O A 4$ is required for carbon utilization, UBP10 is important for nitrogen utilization (Enyenihi and Saunders 2003), UBP15 plays important roles in growth and stress response (Yoshikawa et al. 2011), and UBP14 plays roles in proteasome-mediated proteolysis (Hadari et al. 1992; Amerik et al. 1997; Eisele et al. 2006). Disruption of several $S$. cerevisiae $U B P$ genes, including DOA4, UBP6, UВP8, UBP10 and $U B P 14$, led to increasement of the ubiquitin levels (Amerik et al. 2000), similar to most of the UBP gene disruption mutants of $M$. oryzae. The $U B P$ genes were also studied in other fungi. For example, in Cryptococcus neoformans, UBP5 is likely the major deubiquitinating enzyme-coding gene for stress responses, whose disruption mutant is severely reduced in virulence, capsule formation, melanization, sporulation, and is more sensitive to different stresses (Fang et al. 2012). Our previous study found that deletion of M. oryzae UBP14 resulted in loss of virulence, as well as pleiotropic phenotypic defects such as decrease in colony growth, reduction in conidium and appressorium formation, increase in sensitivity to stresses, and reduction in carbon sources utilization (Wang et al. 2018). Similarly, another study found that the $\Delta M o u b p 4$ mutants also lose their pathogenicity, and are blocked in penetration and invasive growth. The conidia produced by the $\Delta M o u b p 4$ mutants are defective in appressorium formation (Que et al. 2019). In this study, we systemically analyzed functions of the $U B P$ genes in $M$. oryzae, and found some of the $U B P$ genes also play important roles in nutrient utilization, stress responses, mycelium growth, conidium formation, and infection.
In many fungal pathogens, including $M$. oryzae, conidiogenesis is a key step in the infection process. Our result showed the $\Delta u b p 1, \Delta u b p 3, \Delta u b p 4, \Delta u b p 6, \Delta u b p 8$, $\Delta u b p 12, \Delta u b p 13, \Delta u b p 14$ and $\Delta u b p 16$ were significantly reduced in conidiation, suggesting that $U B P$ gene family plays key roles in conidium formation. We also found that the $U B P$ genes may function as both positive and negative regulators in stress responses and nutrient utilization of $M$. oryzae. For example, UBP1, UBP3, $U B P 8, U B P 12$ and $U B P 14$ could be positive regulators in different stress responses of $M$. oryzae, UBP2 and UBP4 were negative regulators in cell wall stress response, and $U B P 1, U B P 2$ and $U B P 4$ were negative regulators in oxidative stress response (Fig. 8). Similar situation was also found in nutrient utilization. UBP4 and UBP14 were positive regulators of nutrient utilization, while $U B P 1$ and $U B P 8$ were negative regulators. These data suggest that de-ubiquitination system function in both positive and negative manners in different cellular processes.

\section{Conclusions}

In this study, we reported that 11 members of the UBP family are involved in the growth, development, stress responses, nutrient utilization, and pathogenicity of $M$. oryzae. Given the importance of these proteins, further investigation of the $U B P$ genes and their respective regulatory networks are required, and they may also serve as putative fungicide targets for disease control.

\section{Methods}

\section{Strains and culture conditions}

The wild-type strain P131 and mutant strains of M. ory$z a e$ were grown at $28^{\circ} \mathrm{C}$ on Oatmeal Tomato Agar (OTA) plates. Mycelia cultured in liquid $\mathrm{CM}$ at $28^{\circ} \mathrm{C}$ were used for extracting genomic DNA, RNA, protein, and isolating protoplasts. Colony growth and conidiation were performed as described previously (Chen et al. 
2014). Conidia harvested from 7-day-old OTA cultures were used for testing virulence and observing infection process. Stress sensitivity of different strains were tested at 5 days after incubation on $\mathrm{CM}$ plates containing 0.1 $\mathrm{mg} / \mathrm{mL}$ CFW, $0.2 \mathrm{mg} / \mathrm{mL}$ CR, $0.005 \%$ SDS, $0.5 \mathrm{M} \mathrm{NaCl}$, or $10 \mathrm{mM} \mathrm{H}_{2} \mathrm{O}_{2}$. Colony diameters were recorded to calculate the growth reduction rates. Carbon source utilization was tested by recording colony diameters of different strains incubated on MM agar plates containing $1 \%$ glucose, $5 \mathrm{mM}$ sodium acetate, $2 \%$ ethanol, or $2 \%$ glycerol as the sole carbon source. Colony diameters were recorded to calculate the growth reduction rates.

\section{Gene deletion transformation}

A split-PCR mediated gene deletion strategy was used to delete the UBP genes (Goswami 2012). Gene replacement construct was generated by respectively fusing 1.5$\mathrm{kb}$ upstream and 1.5-kb downstream flanking sequences with part of the hygromycin segment, and then transformed into protoplasts of the wild type (Chen et al. 2014) (Additional file 3: Table S1). The deletion transformants were selected by using $250 \mu \mathrm{g} / \mathrm{mL}$ hygromycin $B$ (Roche, USA), and verified by PCR-mediated methods (Liu et al. 2018) (Additional file 3: Table S1).

\section{Virulence test and infection process observation}

Conidia suspensions $\left(5 \times 10^{4}\right.$ conidia/mL, in $\mathrm{ddH}_{2} \mathrm{O}$ containing $0.025 \%$ Tween 20) of different strains were sprayed onto one-month-old rice seedlings (Oryza sativa cv. LTH). The inoculated rice seedlings were incubated at $28^{\circ} \mathrm{C}$ with full humidity, and the disease lesions were observed and photographed at 5 days post inoculation [dpi]. For infection process observation, the conidia suspensions $\left(1 \times 10^{5}\right.$ conidia $\left./ \mathrm{mL}\right)$ were dropped onto the barley leaves, and the leaves were then put into a dark chamber with full humidity at $28^{\circ} \mathrm{C}$. Infection processes were observed under a microscope (Nikon Ni90, Japan) at different time points ( $24 \mathrm{hpi}$ and $30 \mathrm{hpi})$.

\section{Expression level analysis of UBP genes}

To examine $U P B$ gene transcript levels at different tissues of $M$. oryzae, samples of mycelia, appressoria and infection hyphae were harvested as previously described (Hendy et al. 2019), and were used to extract total RNA for the RNAseq experiment, from which the expression levels of $U B P$ genes were determined by FPKM (Fragments Per Kilobase of transcript per Million mapped reads) values.

\section{Phenotypic characterization of the ubp mutants}

Mycelial growth of the different strains was measured on OTA plates after $120 \mathrm{~h}$ of incubation at $28^{\circ} \mathrm{C}$. Conidiation were calculated from conidia produced on OTA plates as previously reported (Chen et al. 2014).
Appressoria formation was observed by dropping conidia suspension $\left(1 \times 10^{5}\right.$ conidia/mL) onto a hydrophobic coverslip, then incubating in a moistened chamber at $28^{\circ} \mathrm{C}$. Appressoria formation percentage was calculated under a microscopy with at least 100 conidia per replicate and three replicates per experiment.

\section{Staining assays}

For CFW staining assay, mycelia were harvested from $\mathrm{CM}$ medium and stained with $10 \mu \mathrm{g} / \mathrm{mL}$ CFW (SigmaAldrich, USA) for $10 \mathrm{~min}$ in the dark. Before observation under a fluorescence microscope (Nikon Ni90 microscope, Japan), the samples were rinsed twice with PBS buffer. For DAB staining assay, the barley leaves were inoculated with different strains by dropping with conidia suspension $\left(1 \times 10^{5}\right.$ conidia/mL), then leave samples were stained with $\mathrm{DAB}$ solution $(1 \mathrm{mg} / \mathrm{mL}, \mathrm{pH} 3.8$, Sigma-Aldrich, USA) at $36 \mathrm{hpi}$. After staining for $8 \mathrm{~h}$, the leave samples were de-stained with an ethanol/acetic acid solution (ethanol/acetic acid; 94:4) for $1 \mathrm{~h}$ and observed under an epifluorescence microscope (Nikon Ni90 microscope, Japan).

\section{Ubiquitination level detection}

Total proteins of different strains were extracted as previously reported (Wang et al. 2018), which were subsequently assayed by Western blot analysis with an antiubiquitin as the primary antibody (1:5000, Sigma, USA) and an anti-mouse horseradish peroxidase as the secondary antibody (1:10000, Sigma, USA). Results were visualized with the ECL detection system (Amersham Biosciences, Piscataway, NJ).

\section{Statistical analyses}

All the data analysis was performed using SPSS (Statistical Program for Social Sciences) Version 10.0.

\section{Supplementary information}

Supplementary information accompanies this paper at https://doi.org/10. 1186/s42483-020-00050-1.

\footnotetext{
Additional file 1 Figure $\mathbf{S 1}$. Replacement strategy for M. oryzae UBP family genes through a split-marker approach. White bars represent genomic regions upstream and downstream of the UBP coding sequence that were amplified and fused to segments of the hygromycin phosphotransferase (HYG) cassette.

Additional file $\mathbf{2}$ Figure S2. Verification of the putative deletion mutants of $M$. oryzae UBP family genes. a PCR verification of the flanking sequences beside the replacement fragment by using primer pairs of LCK/HCK-up and RCK/HCK-down. b PCR verification by amplifying the UBP genes in the transformants and the wild-type strain (WT).

Additional file $\mathbf{3}$ Table S1. Primers used in this study.
}

\section{Abbreviations}

CFW: Calcofluor White; CR: Congo Red; DAB: 3,3'-diaminobenzidine; DUB: Deubiquitinating enzyme; OTA: Oatmeal tomato agar; ROS: Reactive oxygen 
species; SDS: Sodium dodecyl sulfate; UBP: Ubiquitin-specific protease; UCH: Ubiquitin C-terminal hydrolase

\section{Acknowledgments \\ Not applicable.}

\section{Authors' contributions}

X-LC conceived and designed the experiments. XC, ZW, YH, CL and HA conducted the experiments. JX provided technical support and edited the manuscript. X-LC and XC wrote the manuscript. All authors read and approved the final manuscript.

\section{Funding}

This work was supported by the Natural Science Foundation of China (Grant 31871909) and the Open Research Fund of State Key Laboratory of Hybrid Rice (Hunan Hybrid Rice Research Center) (2019KF04).

\section{Availability of data and materials}

Not applicable.

\section{Ethics approval and consent to participate}

Not applicable.

\section{Consent for publication}

Not applicable.

\section{Competing interests}

The authors declare that they have no competing interests.

\section{Author details}

${ }^{1}$ The Provincial Key Lab of Plant Pathology of Hubei Province, College of Plant Science and Technology, Huazhong Agricultural University, Wuhan 430070, China. ${ }^{2}$ Department of Agricultural Botany, Faculty of Agriculture (Saba Basha), Alexandria University, Alexandria 21513, Egypt. ${ }^{3}$ State Key Laboratory of Hybrid Rice, Hunan Hybrid Rice Research Center, Changsha 410082, China.

Received: 28 February 2020 Accepted: 31 March 2020

\section{Published online: 07 April 2020}

\section{References}

Amerik AY, Hochstrasser M. Mechanism and function of deubiquitinating enzymes. Biochim Biophys Acta. 2004;1695:189-207.

Amerik AY, Li SJ, Hochstrasser M. Analysis of the deubiquitinating enzymes of the yeast Saccharomyces cerevisiae. Biol Chem. 2000;381(9-10):981-92.

Amerik AY, Swaminathan S, Krantz BA, Wilkinson KD, Hochstrasser M. In vivo disassembly of free polyubiquitin chains by yeast Ubp14 modulates rates of protein degradation by the proteasome. EMBO J. 1997;16:4826-38.

Auesukaree C, Damnernsawad A, Kruatrachue M, Pokethitiyook P, Boonchird C, Kaneko Y, et al. Genome-wide identification of genes involved in tolerance to various environmental stresses in Saccharomyces cerevisiae. J Appl Genet. 2009;50:301-10.

Balakirev MY, Tcherniuk SO, Jaquinod M, Chroboczek J. Otubains: a new family of cysteine proteases in the ubiquitin pathway. EMBO Rep. 2003;4(5):517-22.

Burnett B, Li F, Pittman RN. The polyglutamine neurodegenerative protein ataxin3 binds polyubiquitylated proteins and has ubiquitin protease activity. Hum Mol Genet. 2003;12(23):3195-205.

Chen XL, Shi T, Yang J, Shi W, Gao X, Chen D, et al. N-glycosylation of effector proteins by an alpha-1,3-mannosyltransferase is required for the rice blast fungus to evade host innate immunity. Plant Cell. 2014;26:1360-76.

Crosas B, Hanna J, Kirkpatrick DS, Zhang DP, Tone Y, Hathaway NA, et al. Ubiquitin chains are remodeled at the proteasome by opposing ubiquitin ligase and deubiquitinating activities. Cell. 2006;127:1401-13.

de Jong JC, McCormack BJ, Smirnoff N, Talbot NJ. Glycerol generates turgor in rice blast. Nature. 1997;389:244-5.

Dikic I, Wakatsuki S, Walters KJ. Ubiquitin-binding domains - from structures to functions. Nat Rev Mol Cell Biol. 2009;10(10):659-71.

Dixon KP, Xu JR, Smirnoff N, Talbot NJ. Independent signaling pathways regulate cellular turgor during hyperosmotic stress and appressorium-mediated plant infection by Magnaporthe grisea. Plant Cell. 1999;11:2045-58.
Doelling JH, Yan N, Kurepa J, Walker J, Vierstra RD. The ubiquitin-specific protease UBP14 is essential for early embryo development in Arabidopsis thaliana. Plant J. 2001;27:393-405.

Du L, Li N, Chen L, Xu Y, Li Y, Zhang Y, et al. The ubiquitin receptor DA1 regulates seed and organ size by modulating the stability of the ubiquitinspecific protease UBP15/SOD2 in Arabidopsis. Plant Cell. 2014;26:665-77.

Dudley AM, Janse DM, Tanay A, Shamir R, Church GM. A global view of pleiotropy and phenotypically derived gene function in yeast. Mol Syst Biol. 2005;1:2005.0001. https://doi.org/10.1038/msb4100004.

Eisele F, Braun B, Pfirrmann T, Wolf DH. Mutants of the deubiquitinating enzyme Ubp14 decipher pathway diversity of ubiquitin-proteasome linked protein degradation. Biochem Biophys Res Commun. 2006;350:329-33.

Enyenihi AH, Saunders WS. Large-scale functional genomic analysis of sporulation and meiosis in Saccharomyces cerevisiae. Genetics. 2003;163:47-54.

Fang W, Price MS, Toffaletti DL, Tenor J, Betancourt-Quiroz M, Price JL, et al. Pleiotropic effects of deubiquitinating enzyme Ubp5 on growth and pathogenesis of Cryptococcus neoformans. PLoS One. 2012;7(6):e38326.

Goswami RS. Targeted gene replacement in fungi using a split marker approach. Methods Mol Biol. 2012;835:255-69.

Hadari T, Warms JV, Rose IA, Hershko A. A ubiquitin C-terminal isopeptidase that acts on polyubiquitin chains. Role in protein degradation. J Biol Chem. 1992; 267:719-27.

Hendy AA, Xing J, Chen X, Chen XL. The farnesyltransferase $\beta$-subunit RAM1 regulates localization of RAS proteins and appressorium-mediated infection in Magnaporthe oryzae. Mol Plant Pathol. 2019;20(9):1264-78.

Hershko A, Ciechanover A. The ubiquitin system. Annu Rev Biochem. 1998;67:425-79.

Hu M, Li P, Li M, Li W, Yao T, Wu JW, et al. Crystal structure of a UBP-family deubiquitinating enzyme in isolation and in complex with ubiquitin aldehyde. Cell. 2002;111:1041-54.

Johnston SC, Riddle SM, Cohen RE, Hill CP. Structural basis for the specificity of ubiquitin C-terminal hydrolases. EMBO J. 1999;18(14):3877-87.

Kahana A. The deubiquitinating enzyme Dot4p is involved in regulating nutrient uptake. Biochem Biophys Res Commun. 2001;282:916-20.

Kankanala P, Czymmek K, Valent B. Roles for rice membrane dynamics and plasmodesmata during biotrophic invasion by the blast fungus. Plant Cell. 2007:19:706-24.

Liu C, Li Z, Xing J, Yang J, Wang Z, Zhang H, et al. Global analysis of sumoylation function reveals novel insights into development and appressorium-mediated infection of the rice blast fungus. New Phytol. 2018:219(3):1031-47.

Liu Y, Wang F, Zhang H, He H, Ma L, Deng XW. Functional characterization of the Arabidopsis ubiquitin-specific protease gene family reveals specific role and redundancy of individual members in development. Plant J. 2008;55(5):844-56.

Nanao MH, Tcherniuk SO, Chroboczek J, Dideberg O, Dessen A, Balakirev MY. Crystal structure of human otubain 2. EMBO Rep. 2004;5(8):783-8.

Nijman SMB, Luna-Vargas MPA, Velds A, Brummelkamp TR, Dirac AMG, Sixma TK, et al. A genomic and functional inventory of deubiquitinating enzymes. Cell. 2005;123(5):773-86.

Pickart CM, Eddins MJ. Ubiquitin: structures, functions, mechanisms. Biochim Biophys Acta. 2004;1695:55-72.

Que Y, Xu Z, Wang C, Lv W, Yue X, Xu L, et al. The putative deubiquitinating enzyme MoUbp4 is required for infection-related morphogenesis and pathogenicity in the rice blast fungus Magnaporthe oryzae. Curr Genet. 2019. https://doi.org/10.1007/s00294-019-01049-8.

Scheel H, Tomiuk S, Hofmann K. Elucidation of ataxin-3 and ataxin-7 function by integrative bioinformatics. Hum Mol Genet. 2003;12(21):2845-52.

Thines E, Weber RW, Talbot NJ. MAP kinase and protein kinase Adependent mobilization of triacylglycerol and glycogen during appressorium turgor generation by Magnaporthe grisea. Plant Cell. 2000;12:1703-18.

Wang Z, Zhang H, Liu C, Xing J, Chen XL. A deubiquitinating enzyme Ubp14 is required for development, stress response, nutrient utilization, and pathogenesis of Magnaporthe oryzae. Front Microbiol. 2018:9:769.

Wilkinson KD. Regulation of ubiquitin-dependent processes by deubiquitinating enzymes. FASEB J. 1997;11:1245-56.

Wilson RA, Talbot NJ. Under pressure: investigating the biology of plant infection by Magnaporthe oryzae. Nat Rev Microbiol. 2009;7:185-95.

Wu R, Shi Y, Zhang Q, Zheng W, Chen S, Du L, et al. Genome-wide identification and characterization of the UBP gene family in Moso bamboo (Phyllostachys edulis). Int J Mol Sci. 2019;20(17):4309. 
Xu Y, Jin W, Li N, Zhang W, Liu C, Li C, et al. Ubiquiitin-specific protease14 interacts with ultiraviolet-B insensitive4 to regulate endoreduplication and cell and organ growth in Arabidopsis. Plant Cell. 2016;28:1200-14.

Yan X, Talbot NJ. Investigating the cell biology of plant infection by the rice blast fungus Magnaporthe oryzae. Curr Opin Microbiol. 2016;34:147-53.

Yoshikawa K, Tanaka T, Ida Y, Furusawa C, Hirasawa T, Shimizu H. Comprehensive phenotypic analysis of single-gene deletion and overexpression strains of Saccharomyces cerevisiae. Yeast. 2011;28:349-61.

Zhao J, Zhou H, Zhang M, Gao Y, Li L, Gao Y, et al. Ubiquitin-specific protease 24 negatively regulates abscisic acid signaling in Arabidopsis thaliana. Plant Cell Environ. 2016:39:427-40

Ready to submit your research? Choose BMC and benefit from:

- fast, convenient online submission

- thorough peer review by experienced researchers in your field

- rapid publication on acceptance

- support for research data, including large and complex data types

- gold Open Access which fosters wider collaboration and increased citations

- maximum visibility for your research: over $100 \mathrm{M}$ website views per year

At $\mathrm{BMC}$, research is always in progress.

Learn more biomedcentral.com/submissions 